\title{
Desempeño cognoscitivo y mortalidad en personas mayores de 50 años en México
}

\author{
Enrique Díaz de León González, ${ }^{1}$ Abel Jesús Barragán Berlanga, ${ }^{2}$ \\ Hugo Gutiérrez Hermosillo ${ }^{3}$ y Héctor Cobos Aguilar ${ }^{4}$
}

Forma de citar

Díaz de León González E, Barragán Berlanga AJ, Gutiérrez Hermosillo H, Cobos Aguilar H. Desempeño cognoscitivo y mortalidad en personas mayores de 50 años en México. Rev Panam Salud Publica. 2010;27(5):368-75.

RESUMEN Objetivo. Determinar la asociación entre un bajo desempeño cognoscitivo registrado en 2001 en el Estudio Nacional sobre Salud y Envejecimiento en México (ENASEM), y la mortalidad notificada en 2003 en la segunda vuelta del mismo estudio.

Método. Se seleccionaron sujetos de 50 o más años de edad que hubiesen completado las secciones de la encuesta de 2001 sobre ejercicios cognoscitivos, estado de salud, y funcionalidad y ayuda para el desempeño de actividades cotidianas. El desempeño cognoscitivo fue evaluado en 2001 utilizando una versión reducida del Examen Cognoscitivo Transcultural (escala de 0 a 80 puntos). En 2003 se realizó el seguimiento de los entrevistados y se registró su supervivencia o mortalidad.

Resultados. Los grupos de entrevistados con puntaje menor a 40 notificaron una supervivencia acumulada de 96,1\% y una media de 26,49 meses de supervivencia (IC 95\% 26,4126,57), mientras que los de 40 o más puntos informaron una supervivencia acumulada de $98,7 \%$ y una media de supervivencia de 28,76 meses (IC 95\% 28,68-28,85) $\left(\log\right.$ Rank $\chi^{2}=59,230$ $\mathrm{P}<0,001)$. Un puntaje menor a 40 en la evaluación cognoscitiva se asoció con un riesgo relativo de muerte de 1,863 (IC 95\% 1,30-2,65) en el análisis multivariado. También se asociaron con tasas más altas de mortalidad una mayor edad, autoinforme de diabetes, cáncer, haber fumado alguna vez, recibir ayuda en al menos una actividad instrumentada de la vida diaria y tener un mayor puntaje en la escala de depresión.

Conclusiones. Un puntaje menor a 40 en la versión abreviada del Examen Cognoscitivo Transcultural se asoció independientemente con un incremento en las tasas de mortalidad dentro de los dos años siguientes.

Palabras clave Envejecimiento; mortalidad; salud del adulto; salud del anciano; salud mental; actividades cotidianas; México.

Programa Multicéntrico de Residencias Médicas, Departamento de Geriatría, Hospital San José Tec de Monterrey-Servicios de Salud de Nuevo León, Hospital Metropolitano Dr. Bernardo Sepúlveda. Departamento de Posgrado, Universidad Juárez del Estado de Durango, México. La correspondencia debe dirigirse a Enrique Díaz de León González, Ave. López Mateos y Nogalar \# 4600, Col. Bosques de Nogalar, San Nicolás de los Garza, Nuevo León, México. Correo electrónico: edleon20@hotmail.com

2 Programa Multicéntrico de Residencias Médicas, Departamento de Geriatría, Hospital San José Tec de Monterrey-Servicios de Salud de Nuevo León,
La transición demográfica y epidemiológica asociada al aumento en la espe-

Hospital Metropolitano Dr. Bernardo Sepúlveda, Nuevo León, México.

3 Departamento de Geriatría, Hospital General de Zona No. 17, Instituto Mexicano del Seguro Social, Monterrey, Nuevo León, México. Departamento de Posgrado, Universidad Juárez del Estado de Durango, México.

4 División de Ciencias de la Salud, Universidad de Monterrey, Nuevo León, México. ranza de vida ha tenido implicaciones importantes para los sistemas de salud en todo el mundo. Una de ellas es el envejecimiento poblacional. En México, entre 2005 y 2050 la población de adultos mayores aumentará en alrededor de 26 millones, aunque más de $75 \%$ de este incremento ocurrirá a partir de 2020. Debido a este acelerado crecimiento, se estima que la población de 60 años o más, 
que a la fecha representa casi uno de cada 13 mexicanos $(7,6 \%)$, en 2030 representará uno de cada seis $(17,1 \%)$ y en 2050 más de uno de cada cuatro $(27,7 \%)$. La media de edad de la población, que en la actualidad es de 28 años, ascenderá a 37 y 43 años en 2030 y 2050, respectivamente (1). Es de esperar que este fenómeno genere un aumento de las enfermedades asociadas con la edad, entre ellas el deterioro cognoscitivo, una condición que afecta de manera directa a la calidad de vida de la población adulta mayor, lo que determinará un mayor uso de los servicios de salud y repercutirá en los costos directos e indirectos de su atención.

Hoy por hoy el deterioro cognoscitivo persiste como un concepto mal definido y poco consensuado, que refleja una disminución del rendimiento de al menos una de las capacidades mentales siguientes: memoria, orientación, pensamiento abstracto, lenguaje, juicio y razonamiento, capacidad para el cálculo y habilidad constructiva, capacidad de aprendizaje y habilidad visuoespacial. Presupone un nivel cognoscitivo previo superior al actual, declive o merma respecto al nivel de funcionamiento habitual, y pérdida parcial o general de las capacidades previamente adquiridas, lo que no siempre resulta demostrable (2).

Hasta la fecha, la frontera entre el funcionamiento cognoscitivo normal y el deterioro, con y sin demencia, aún no está bien delimitada. Según lo propuesto por Petersen, un estadio entre la demencia y el funcionamiento cognoscitivo normal constituiría el deterioro cognoscitivo leve (3). Las personas con "deterioro cognoscitivo leve" revelan problemas en la memoria ( $u$ otros dominios cognoscitivos) más allá de lo esperado para un envejecimiento normal (3). No obstante, esta definición implica que se entiende lo que constituyen las funciones cognoscitivas normales para la edad, lo cual no está muy claro aún. Algunos autores sostienen que una pérdida de las habilidades cognoscitivas es parte de un envejecimiento normal, mientras que otros opinan que, en ausencia de enfermedad, tales habilidades no deberían verse afectadas por la edad (3). Si bien el término "normal" se utiliza extendidamente como concepto operativo, dicho término tiene varias definiciones. Desde el punto de vista neuropsicológico, el estado normal se determina por comparación con una norma que representa lo común y lo estándar (4). Sin embargo, cabe preguntarse: ¿común o estándar para qué? o ¿para quiénes?, pues las normas y estándares tienden a variar $-\mathrm{y}$ de hecho lo hacen- según las distintas poblaciones.

Existen otras tres definiciones de normalidad y son las que se emplean en epidemiología clínica (5). La primera define lo normal como aquello más común o usual y anormal como aquello menos común o inusual (5). Se trata de una definición estadística empleada en pruebas cognoscitivas y basada en la frecuencia con que se dan ciertas características en una población determinada. También considera normal a toda medición que se encuentra entre dos desviaciones estándar de la media, siempre que siga una distribución normal, o a aquellos casos de distribución no normal que se encuentran entre los percentiles 2,5 y 97,5 (5). Esta versión tiene sus limitaciones, ya que no siempre ni necesariamente existe relación entre posición en percentiles extremos y enfermedad. La segunda definición es la que considera anormal aquello asociado con enfermedad, discapacidad o muerte (5). Esta definición es la que plantea un enfoque más clínico y por ende la que más interesa para la toma de decisiones en medicina. La tercera definición contempla lo anormal como algo susceptible de tratamiento (5), es decir inclinándose a considerar las situaciones clínicas asintomáticas como anormales cuando su tratamiento podría llevar a un mejor resultado clínico (p. ej. mayor supervivencia o mejor calidad de vida).

Sin embargo, y pese a las definiciones anteriores, existe un uso muy extenso del término "deterioro cognoscitivo leve," en referencia a un estadio intermedio entre un envejecimiento cerebral normal y uno patológico, existiendo actualmente consenso en que se trata de un estado de aptitud cognoscitiva baja. Aun así, no hay un proceso acordado para caracterizar esta condición de manera homogénea y uniforme (6-8). Hoy en día el término "deterioro cognoscitivo leve" es usado para diagnosticar personas que tienen signos evidentes de deterioro o bajo desempeño cognoscitivo en pruebas neuropsicológicas, y que no reúnen criterios diagnósticos de demencia. No obstante, la definición empleada en la población es de carácter estadístico y se ajusta según edad y años de escolaridad (9), además de que en el caso de la demencia se requiere una falla en el funcio- namiento de las actividades diarias. Hay datos que demuestran que los bajos desempeños cognoscitivos se asocian con una mortalidad y morbilidad importantes, que pueden llegar a ser hasta dos veces más elevadas en las personas con dichos trastornos en comparación con quienes no los padecen (10-17).

México cuenta con la base de datos del ENASEM $^{5}$, una encuesta sobre los procesos de envejecimiento y el peso de las enfermedades que incluye, entre otros componentes, la evaluación neuropsicológica a través de la versión reducida del Examen Cognoscitivo Transcultural, y que registra datos de la primera vuelta realizada en 2001 y de la segunda en 2003, con medición de la variable mortalidad en esta última. A partir de esta base de datos se han publicado numerosos informes relacionados con el estado de salud del anciano, aun cuando todavía no se ha evaluado el desempeño cognoscitivo y su asociación con la mortalidad. En tal sentido, el objetivo del presente trabajo fue precisamente determinar la asociación entre un bajo desempeño cognoscitivo en la versión reducida del Examen Cognoscitivo Transcultural aplicado en 2001 en el ENASEM y la mortalidad entre 2001 y 2003 notificada en la segunda vuelta de dicha encuesta.

\section{MATERIALES Y MÉTODOS}

Para la realización del presente trabajo se empleó la base de datos del ENASEM, que es un estudio prospectivo efectuado en sujetos mexicanos nacidos antes de 1951, así como en sus parejas. El diseño metodológico fue realizado por el Centro de Estudios de Población de la Universidad de Pensilvania, el Centro de Investigaciones de Población de la Universidad de Maryland y el Centro de Demografía y Ecología de la Universidad de Wisconsin, mientras que el trabajo de campo estuvo a cargo del Instituto Nacional de Estadística, Geografía e Informática (INEGI) de México. En el estudio se recoge información relacionada con aspectos tales como dinámica de la salud, estructura familiar y transferencias intergeneracionales, comportamientos migratorios y diferencias socioeconómicas según ingresos y posesión de bienes. La muestra es representativa del componente no institucionalizado de la pobla-

\footnotetext{
Estudio Nacional sobre Salud y Envejecimiento en México.
} 
ción mayor de 50 años en el año 2000. La recolección de los datos concluyó en el verano de 2001, y en 2003 se realizó una segunda vuelta del estudio. En 2001 participaron 15402 sujetos, mientras que en 2003 se entrevistó a 14386 participantes, de los cuales 13620 correspondieron al seguimiento de los encuestados en 2001. Los factores de ponderación se definieron de acuerdo con cuatro criterios: a) por hogar, b) por sujetos de 50 años o más con pareja de 50 años o más en el mismo hogar, c) por sujetos de 50 años o más sin pareja y d) por sujetos menores de 50 años con pareja de 50 años o más en el mismo hogar. Se seleccionaron todos los sujetos de 50 años o más que completaron en su totalidad las secciones de ejercicios cognoscitivos, estado de salud, funcionalidad y necesidad de ayuda en 2001, a quienes se les realizó una visita de seguimiento en 2003 donde se determinó si aún vivían. Todos aquellos sujetos que presentaron valores perdidos en las variables estudiadas fueron excluidos del análisis.

\section{Variables analizadas}

En la evaluación cognoscitiva se aplicó la versión reducida del Examen Cognoscitivo Transcultural. Esta prueba tiene una puntuación máxima de 80 puntos e incluye la evaluación de las siguientes funciones cognoscitivas: a) memoria verbal primaria (repetición de ocho palabras, con puntaje de 0 a 8); b) atención selectiva (detección visual de un estímulo blanco, con puntaje de 0 a 60); c) memoria verbal secundaria (evocación de las ocho palabras, con puntaje de 0 a 8); d) praxias visoconstructivas (copia de dos figuras geométricas, con puntaje de 0 al 2); y e) memoria visual (evocación de dos figuras geométricas, puntaje de 0 a 2). La prueba tiene una sensibilidad de $100 \%$ y una especificidad de $83 \%$ para pacientes con demencia (9). Se dicotomizó el puntaje total (desempeño) de la evaluación cognoscitiva en menos de 40 puntos y 40 ó más y en función de esta variable se crearon los grupos expuesto y no expuesto, respectivamente, constituyéndose así la variable del desempeño cognoscitivo. Se incluyeron además para su análisis posibles variables de confusión: género, edad, escolaridad, autoinforme de hipertensión o presión alta, diabetes o nivel de azúcar alto en sangre, cáncer, enfermedad pulmonar, enfermedad cardíaca (ataque al corazón), enfermedad cerebral (embolia, derrame cerebral o isquemia cerebral transitoria), presencia/ausencia de pérdida de peso de $5 \mathrm{~kg}$ o más, artritis o reumatismo, infección renal o hepática, tuberculosis, neumonía, fractura ósea, uso de lentes, calidad de la visión, uso de dispositivo auditivo, dolor, tabaquismo en alguna ocasión, tabaquismo actual, consumo de bebidas alcohólicas, presencia/ausencia de caídas en los últimos dos años y autopercepción de salud.

Para su registro, los sujetos respondieron a la pregunta sobre presencia/ ausencia de diagnóstico médico de las diferentes enfermedades. Cabe destacar que se incluyeron síntomas físicos como hinchazón de pies, dificultad para respirar, mareos o desmayos, sed intensa, fatiga o cansancio, jadeo, tos o producción de flema, dolor de piernas, pérdida involuntaria de orina y ausencia de alguna extremidad. Se adoptó asimismo la variable depresión, que corresponde a la puntuación total (0-9) obtenida por cada sujeto en un cuestionario de nueve preguntas sobre síntomas depresivos, el cual fue posteriormente validado (18). También se incluyó evaluación funcional por medio de la proporción de pacientes que tienen dificultad o que requieren ayuda en por lo menos una actividad básica de la vida diaria (bañarse, vestirse, ir al baño, desplazarse, alimentarse y ser continente), así como la proporción de pacientes con dificultad o requieren ayuda en por lo menos una actividad instrumentada de la vida diaria (preparar comida caliente, comprar víveres, tomar sus medicamentos y manejar su dinero). Se consideraron como valores perdidos aquellos sin respuesta, que no contestaron o respondieron "no sé" en cada una de las variables analizadas. Todos los sujetos que participaron en el estudio dieron su consentimiento informado al momento de la entrevista.

\section{Análisis estadístico}

El análisis fue realizado por medio del método de casos completos. Los participantes fueron caracterizados mediante estadística descriptiva. Para las variables numéricas se usaron medidas de tendencia central con su respectiva medida de dispersión y con previa comprobación de su normalidad por medio de análisis exploratorio de datos y con la prueba de Kolmogorov-Smirnov. Para las variables cualitativas se usaron frecuencias absolutas y porcentajes. Se emplearon las prue- bas de la ji al cuadrado para determinar diferencia de proporciones, así como la prueba U de Mann-Whitney para demostrar diferencia entre las variables cuantitativas. Se tomó como nivel de significación estadística un valor de $P<0,05$. Se midió el grado de asociación de las variables cualitativas con riesgo relativo con su intervalo de confianza al 95\% y análisis multivariado de las variables significativas con el modelo de regresión de Cox. Se realizó análisis de supervivencia por medio del método de Kaplan-Meier y se comparó la supervivencia con la prueba Log-Rank (Mantel-Cox).

\section{RESULTADOS}

\section{Características de los participantes}

De los 12691 participantes de 50 años o más a quienes se les realizó visita de seguimiento y en quienes se corroboró su estatus de vida o muerte en 2003, únicamente 8797 (69\%) contaban con datos completos de todas las variables a analizar y pasaron a constituir la muestra de estudio: 4881 y 3916 integraron los grupos expuesto y no expuesto, respectivamente. Los sujetos con puntaje menor a 40 (cuadro 1) tenían mayor edad, menos escolaridad y peor autopercepción de salud; en su mayoría eran mujeres; sufrían con mayor frecuencia hipertensión, diabetes, enfermedad cerebrovascular, artritis o reumatismo, infección renal o hepática, neumonía, caídas y fracturas, problemas de visión (uso de lentes) y dolor crónico; tenían historial de tabaquismo con más frecuencia; consumían alcohol con menos frecuencia; y padecían más a menudo hinchazón de pies, dificultad para respirar, mareos o desmayos, sed intensa, fatiga o cansancio, jadeo, tos o producción de flema, dolor de piernas, pérdida involuntaria de orina, ausencia de alguna extremidad, pérdida de peso de $5 \mathrm{~kg}$ o más, dependencia en al menos una actividad básica e instrumentada de la vida diaria y mayor puntaje en la escala de depresión.

\section{Supervivencia}

Con una media global de seguimiento de 28,5 meses, se registraron 191 muertes en el grupo con puntaje menor a 40, y 49 en el grupo de 40 o más puntos, con una supervivencia acumulada de $96,1 \%$ en el grupo con puntaje menor a 40 y $98,7 \%$ en el grupo de 40 o más puntos. La media de 
CUADRO 1. Variables demográficas y de salud de los entrevistados en el Estudio Nacional sobre Salud y Envejecimiento en México, según el puntaje obtenido en una versión reducida del Examen Cognoscitivo Transcultural (escala de 0 a 80 puntos), México, 2001

\begin{tabular}{|c|c|c|c|c|c|}
\hline \multirow[b]{3}{*}{ Variable } & \multicolumn{4}{|c|}{ Puntaje } & \multirow[b]{3}{*}{$p^{a}$} \\
\hline & \multicolumn{2}{|c|}{$<40(n=4881)$} & \multicolumn{2}{|c|}{$\geq 40(n=3916)$} & \\
\hline & No. & $\%$ & No. & $\%$ & \\
\hline \multicolumn{6}{|l|}{ Variables demográficas } \\
\hline Educación en años (mediana) & \multicolumn{2}{|c|}{$\begin{aligned} 63 & (56-70) \\
3 & (0-6)\end{aligned}$} & \multicolumn{2}{|c|}{$\begin{aligned} 56 & (56-32) \\
6 & (3-9)\end{aligned}$} & $<0,001$ \\
\hline Sexo masculino & 2083 & 43 & 1880 & 48 & $<0,001$ \\
\hline \multicolumn{6}{|l|}{ Antecedentes de importancia } \\
\hline Enfermedad respiratoria & 315 & 7 & 243 & 6 & 0,63 \\
\hline Ataque al corazón & 150 & 3 & 119 & 3 & 0,93 \\
\hline Enfermedad cerebrovascular & 134 & 3 & 53 & 1 & $<0,001$ \\
\hline Artritis o reumatismo & 1107 & 23 & 705 & 18 & $<0,001$ \\
\hline Infección renal o hepática & 543 & 11 & 381 & 10 & 0,03 \\
\hline Tuberculosis & 17 & 0 & 12 & 0 & 0,73 \\
\hline Neumonía & 86 & 2 & 41 & 1 & 0,01 \\
\hline Sí & 1320 & 27 & 1484 & 38 & \\
\hline No & 3083 & 63 & 2127 & 54 & \\
\hline Nunca & 478 & 10 & 305 & 8 & \\
\hline \multicolumn{6}{|l|}{ Síntomas clínicos } \\
\hline Dolor & 2158 & 44 & 1434 & 37 & $<0,001$ \\
\hline Hinchazón de los pies & 1429 & 29 & 991 & 25 & $<0,001$ \\
\hline Dificultad para respirar & 741 & 15 & 476 & 12 & $<0,001$ \\
\hline Mareos o desmayos & 1274 & 26 & 768 & 20 & $<0,001$ \\
\hline Sed intensa & 913 & 19 & 565 & 14 & $<0,001$ \\
\hline Fatiga o cansancio & 1387 & 28 & 844 & 22 & $<0,001$ \\
\hline Jadeo, tos o producción de flema & 927 & 19 & 646 & 17 & 0,002 \\
\hline Dolor en las piernas & 2269 & 47 & 1483 & 38 & $<0,001$ \\
\hline Pérdida involuntaria de orina & 444 & 9 & 224 & 6 & $<0,001$ \\
\hline Falta de una extremidad & 73 & 2 & 40 & 1 & $<0,001$ \\
\hline Recibe ayuda en 1 ó más $A B V D^{b}$ & 107 & 2 & 29 & 1 & $<0,001$ \\
\hline Recibe ayuda en 1 ó más AIVDc & 303 & 6 & 96 & 3 & $<0,001$ \\
\hline Puntaje en escala de depresión (mediana) & 3 & $(2-6)$ & 2 & $(1-5)$ & $<0,001$ \\
\hline \multicolumn{6}{|l|}{ Variables de los sentidos } \\
\hline Uso de lentes & 1708 & 35 & 2167 & 55 & $<0,001$ \\
\hline Calidad de la visión & & & & & $<0,001$ \\
\hline Excelente & 106 & 2 & 158 & 4 & \\
\hline Muy buena & 351 & 7 & 446 & 11 & \\
\hline Buena & 1989 & 41 & 1910 & 49 & \\
\hline Regular & 1927 & 40 & 1197 & 31 & \\
\hline Mala & 498 & 10 & 204 & 5 & \\
\hline Legalmente ciego & 10 & 0 & 1 & 0 & \\
\hline Dispositivo auditivo & 69 & 1 & 43 & 1 & 0,19 \\
\hline
\end{tabular}

a Los datos presentan las medianas (amplitud intercuantílica) o frecuencias absolutas y sus porcentajes, y fueron comparados con las pruebas $U$ de Mann-Whitney y de la ji al cuadrado respectivamente.

${ }^{b}$ ABVD: Actividades básicas de la vida diaria.

${ }^{c}$ AIVD: Actividades instrumentadas de la vida diaria.

supervivencia en meses fue de 26,49 (IC $95 \% 26,41-26,57)$ en el grupo con puntaje menor a 40 y 28,76 (IC $95 \%$ 28,68-28,85) en el grupo de 40 o más puntos (figura 1), Log Rank $\chi^{2}=59,230, P<0,001$.

\section{Factores asociados a mortalidad}

Un puntaje menor a 40 puntos en la evaluación cognoscitiva se asoció con un riesgo relativo de muerte de 3,12 (IC
95\%: 2,29-4,26) en el análisis bivariante y un riesgo relativo de 1,86 (IC 95\% 1,302,65 ) en el multivariante. También se asociaron con mortalidad una mayor edad, autoinforme de diabetes, cáncer, 
FIGURA 1. Curvas de supervivencia al año 2003 de acuerdo al puntaje obtenido en el Examen Cognoscitivo Transcultural aplicado en 2001 en el Estudio Nacional sobre Salud y Envejecimiento en México (ENASEM)

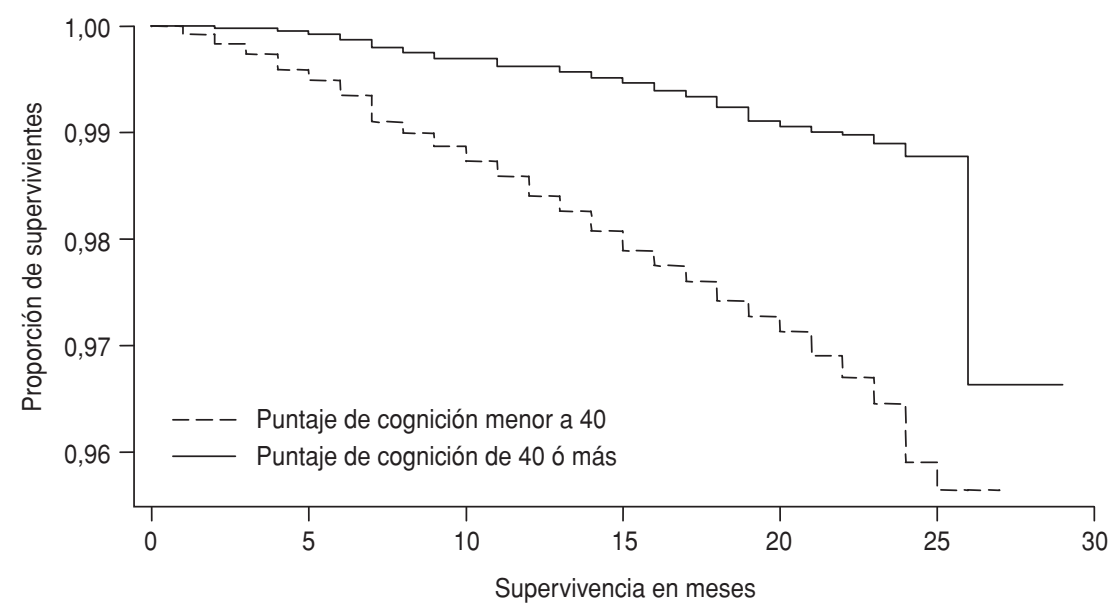

haber fumado alguna vez, recibir ayuda en al menos una actividad instrumentada de la vida diaria y mayor puntaje en la escala de depresión (cuadros 2 y 3).

\section{DISCUSIÓN}

El objetivo del presente análisis fue determinar la asociación entre un puntaje menor a 40 en el examen cognoscitivo transcultural y la mortalidad, aunque este punto de corte incluye sujetos con y sin deterioro cognoscitivo, de acuerdo a definiciones operativas publicadas previamente (9).

Al revisar las características clínicas de los integrantes de los grupos, se encontró que los del grupo expuesto, es decir, con puntaje menor a 40, presentaron más comorbilidades y síntomas físicos. Sin embargo, al ser analizados con el modelo de regresión de Cox incluyendo estas variables asociadas, se halló que los puntajes por debajo de 40 en la evaluación cognoscitiva se vinculan, de manera independiente, con un incremento en la mortalidad a dos años.

Los resultados del presente estudio coinciden en resaltar la asociación entre los valores bajos en pruebas cognoscitivas y la mortalidad publicados por otros autores en distintas poblaciones (10-17, 19). Una revisión sistemática realizada por Batty y colaboradores mostró una relación inversa entre el coeficiente intelectual en adultos jóvenes y la mortalidad (17), que aunque no es comparable con la población mexicana aquí estudiada, confirma dicha asociación independientemente de la edad de los parti- cipantes. Es importante señalar que para el análisis se incluyeron sujetos de 50 años o más, con y sin deterioro cognoscitivo. Se decidió incluir sujetos a partir de los 50 años porque después de esta edad se observa una disminución de las habilidades cognoscitivas, que se hace todavía más evidente después de los 60 años $(20,21)$. Por otro lado, de los trabajos referidos solo Nguyen y colaboradores (19) incluyeron una población similar a la de México -mexicanoestadounidenses de los estados de Arizona, California, Colorado, Nuevo México y Texas-, quienes observaron que aun un deterioro leve en los puntajes del examen mínimo del estado mental se asoció con un incremento en la mortalidad. En un estudio realizado en la ciudad de México por Arauz y colaboradores en el que incluyeron a 734 sujetos de entre 55 y 90 años de edad, evaluados cognitivamente mediante el Examen Mínimo del Estado Mental, con un seguimiento promedio de 3,2 años, se encontró que un deterioro cognoscitivo moderado o severo se asocia con un incremento en la mortalidad, algo que no ocurre con el deterioro leve (22). Una posible explicación de este hallazgo podría ser que se requería un tamaño de muestra mayor para demostrar dicha asociación. Otro trabajo realizado en la ciudad de México por Gutiérrez Robledo y colaboradores (23), en el que se estudiaron 2544 sujetos, evaluados cognoscitivamente con el Examen Mínimo del Estado Mental, reveló un aumento en la mortalidad entre los participantes con desempeños cognoscitivos bajos, in- cluyendo valores considerados normales bajos (23).

La asociación encontrada entre bajo desempeño cognoscitivo y mortalidad es relevante ya que persistió después de haber sido controlada estadísticamente con las características sociodemográficas, puntaje de depresión, funcionalidad y condiciones médicas. Se considera que en términos generales el funcionamiento cognoscitivo está determinado por el estado de salud global (24) y su asociación con la mortalidad podría deberse a esa relación, además de ser síntoma de una amplia gama de enfermedades cerebrovasculares y otras comorbilidades (25) no evaluadas en el presente estudio. Y si bien es probable que condiciones médicas severas medien la asociación entre deterioro cognoscitivo y mortalidad, también lo es que sean un predictor significativo de mortalidad (25).

Aunque en este estudio la escolaridad no fue predictor de mortalidad una vez aplicado el análisis multivariante, tampoco se puede descartar porque la razón de no serlo bien podría deberse al pequeño tamaño de la muestra. De hecho, numerosas publicaciones han mostrado que la baja escolaridad se asocia con el desarrollo de la enfermedad de Alzheimer, así como con bajo nivel socioeconómico, estado nutricional pobre, poco o limitado acceso a los sistemas de salud e inclusive a hábitos como el tabaquismo y la falta de ejercicio $(26,27)$. Más aún, la escolaridad alta se ha propuesto como una constructora de reserva cognoscitiva que protege el cerebro de los procesos degenerativos (26-29).

Una explicación adicional de los resultados del presente estudio podría ser que el bajo desempeño cognoscitivo a menudo se asocia con un nivel socioeconómico bajo - no incluido en este análisis- y que los pacientes en este nivel pudieran tener dificultades para reconocer síntomas de enfermedad, para adherirse a su tratamiento médico e incorporarse a actividades promotoras de salud $(30,31)$, como podría ser el caso de la población mexicana, en la cual el nivel socioeconómico bajo es predominante.

Otras limitaciones del presente análisis se relacionan con las condiciones médicas de la población estudiada, ya que son autoinformes acerca del estado de salud, aun cuando varios estudios han encontrado consistencia con las enfermedades registradas en los archivos médicos $(32,33)$. Asimismo, la pérdida de 
CUADRO 2. Variables demográficas y de salud de los participantes en el Estudio Nacional sobre Salud y Envejecimiento en México (2001), en función de su muerte o supervivencia a los dos años, México, 2001-2003

\begin{tabular}{|c|c|c|c|c|c|c|c|}
\hline \multirow[b]{2}{*}{ Variable } & \multicolumn{2}{|c|}{$\begin{array}{l}\text { Murieron } \\
(n=240)\end{array}$} & \multicolumn{2}{|c|}{$\begin{array}{c}\text { Vivieron } \\
(n=8557)\end{array}$} & \multirow[b]{2}{*}{$P^{a}$} & \multirow[b]{2}{*}{$\mathrm{RR}^{\mathrm{b}}$} & \multirow[b]{2}{*}{$\operatorname{IC} 95 \%{ }^{\mathrm{C}}$} \\
\hline & No. & $\%$ & No. & $\%$ & & & \\
\hline Edad en años (mediana) & \multirow{2}{*}{\multicolumn{2}{|c|}{$\begin{array}{c}69(61-76) \\
3(0-6)\end{array}$}} & \multirow{2}{*}{\multicolumn{2}{|c|}{$\begin{array}{c}59(54-66) \\
4(1-6)\end{array}$}} & $<0,001$ & $N A^{d}$ & $N A^{d}$ \\
\hline Educación en años (mediana) & & & & & $<0,001$ & NA & $N A^{d}$ \\
\hline Sexo masculino & 123 & 51 & 3840 & 45 & 0,05 & 1,28 & $0,99-1,64$ \\
\hline \multicolumn{8}{|l|}{ Antecedentes de importancia } \\
\hline Cáncer & 9 & 4 & 162 & 2 & 0,018 & 1,96 & $1,02-3,75$ \\
\hline Enfermedad respiratoria & 24 & 10 & 534 & 6 & 0,018 & 1,64 & $1,08-2,47$ \\
\hline Ataque al corazón & 18 & 8 & 251 & 3 & $<0,001$ & 2,57 & $1,61-4,09$ \\
\hline Enfermedad cerebrovascular & 10 & 4 & 177 & 2 & 0,026 & 2,00 & $1,08-3,70$ \\
\hline Artritis o reumatismo & 47 & 20 & 1765 & 21 & 0,694 & 0,93 & $0,68-1,28$ \\
\hline Infección renal o hepática & 40 & 17 & 884 & 10 & 0,002 & 1,70 & $1,22-2,37$ \\
\hline Tuberculosis & 3 & 1 & 26 & 0 & 0,012 & 3,82 & $1,30-11,25$ \\
\hline Consume bebidas alcohólicas & & & & & 0,03 & & \\
\hline Sí & 61 & 25 & 2743 & 32 & 0,99 & 1,00 & $0,59-1,71$ \\
\hline No & 162 & 68 & 5048 & 59 & 0,15 & 1,43 & $0,87-2,35$ \\
\hline Nunca & 17 & 7 & 766 & 9 & & 1 & \\
\hline \multicolumn{8}{|l|}{ Síntomas clínicos } \\
\hline Dolor & 116 & 48 & 3476 & 41 & 0,017 & 1,35 & $1,05-1,74$ \\
\hline Hinchazón de los pies & 87 & 36 & 2333 & 27 & 0,002 & 1,49 & $1,15-1,94$ \\
\hline Dificultad para respirar & 52 & 22 & 1165 & 14 & $<0,001$ & 1,72 & $1,27-2,32$ \\
\hline Mareos o desmayos & 66 & 28 & 1976 & 23 & 0,111 & 1,25 & $0,94-1,65$ \\
\hline Sed intensa & 61 & 25 & 1417 & 17 & $<0,001$ & 1,68 & $1,26-2,24$ \\
\hline Fatiga o cansancio & 81 & 34 & 2150 & 25 & 0,002 & 1,49 & $1,15-1,95$ \\
\hline Jadeo, tos o producción de flema & 71 & 30 & 1502 & 18 & $<0,001$ & 1,92 & $1,47-2,53$ \\
\hline Dolor en las piernas & 113 & 47 & 3639 & 43 & 0,159 & 1,19 & $0,93-1,53$ \\
\hline Mala & 74 & 31 & 1213 & 14 & & 1 & \\
\hline Al menos una caída & 115 & 48 & 3119 & 36 & $<0,001$ & 1,58 & $1,23-2,03$ \\
\hline Recibe ayuda en 1 ó más $A B V D^{f}$ & 20 & 8 & 116 & 1 & $<0,001$ & 5,78 & $3,78-8,85$ \\
\hline Recibe ayuda en 1 ó más AIVDg & 39 & 16 & 360 & 4 & $<0,001$ & 4,08 & $2,94-5,66$ \\
\hline Puntaje escala de depresión (mediana) & 4 & $(2,5-7)$ & 3 & $(1-5)$ & $<0,001$ & & \\
\hline \multicolumn{8}{|l|}{ Variables de los sentidos } \\
\hline Uso de lentes & 111 & 46 & 3764 & 44 & 0,486 & 1,09 & $0,85-1,40$ \\
\hline Calidad de la visión & & & & & 0,001 & & \\
\hline Excelente & 2 & 1 & 262 & 3 & 0,009 & 0,08 & $0,01-0,85$ \\
\hline Muy Buena & 15 & 6 & 782 & 9 & 0,19 & 0,21 & $0,03-1,43$ \\
\hline Buena & 102 & 43 & 3797 & 44 & 0,25 & 0,29 & $0,04-1,88$ \\
\hline Regular & 85 & 35 & 3039 & 36 & 0,26 & 0,28 & $0,05-1,96$ \\
\hline Mala & 35 & 15 & 667 & 8 & 0,436 & 0,55 & $0,08-3,65$ \\
\hline Legalmente ciego & 1 & 0 & 10 & 0 & & 1 & \\
\hline Dispositivo auditivo & 1 & 0 & 111 & 1 & 0,23 & 0,32 & $0,04-2,29$ \\
\hline
\end{tabular}

a Los datos presentan las medianas (amplitud intercuantílica) o frecuencias absolutas y sus porcentajes, y fueron comparados con las pruebas $U$ de Mann-Whitney y de la ji al cuadrado respectivamente.

${ }^{\mathrm{b}} \mathrm{RR}$ : riesgo relativo.

c IC95\%: intervalo de confianza de $95 \%$.

${ }^{\mathrm{d}} \mathrm{NA}$ : No aplicable.

${ }^{e}$ ECTC: Examen Cognoscitivo Transcultural.

${ }^{f}$ ABVD: actividades básicas de la vida diaria.

${ }^{9}$ AIVD: actividades instrumentadas de la vida diaria. 
CUADRO 3. Variables clínicas y demográficas de los participantes en el Estudio Nacional sobre Salud y Envejecimiento en México (2001) y su asociación con muerte a los dos años en el análisis multivariante, México, 2001-2003

\begin{tabular}{|c|c|c|c|}
\hline Variable & $\mathrm{RR}^{\mathrm{a}}$ & IC95\% & $P^{c}$ \\
\hline \multicolumn{4}{|l|}{ Variables demográficas } \\
\hline Edad en años & 1,065 & $1,05-1,08$ & $<0,001$ \\
\hline \multicolumn{4}{|l|}{ Antecedentes de importancia } \\
\hline Diabetes & 1,79 & $1,32-2,41$ & $<0,001$ \\
\hline Cáncer & 2,153 & $1,09-4,23$ & 0,026 \\
\hline \multicolumn{4}{|l|}{ Variables de estilo de vida } \\
\hline Alguna vez fumó & 1,344 & $1,005-1,797$ & 0,046 \\
\hline \multicolumn{4}{|l|}{ Variables geriátricas } \\
\hline Puntaje ECTC ${ }^{d}<40$ & 1,863 & $1,30-2,66$ & 0,001 \\
\hline Recibe ayuda en 1 o más AIVDe & 1,596 & $1,04-2,43$ & 0,031 \\
\hline Puntaje en escala de depresión & 1,071 & $1,01-1,13$ & 0,02 \\
\hline
\end{tabular}

a $\mathrm{RR}$ : riesgo relativo.

b IC95\%: intervalo de confianza de $95 \%$.

${ }^{c}$ El análisis multivariado se realizó con el modelo de regresión de Cox, método Introducir.

d ECTC: Examen Cognoscitivo Transcultural.

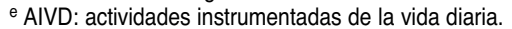

sujetos durante el seguimiento y el análisis de casos completos podrían haber influido sobre los resultados del estudio y producir sesgo de selección (34). Otra limitante es que existen algunas variables de confusión que no se midieron y que son conocidas como factores de riesgo para mortalidad a 5 años (35), así como tampoco se midieron las relacionadas con la fragilidad, descritas por Fried (36).
La principal fortaleza del presente análisis es su aplicabilidad clínica a nivel poblacional, ya que emplea mediciones que se pueden realizar en el consultorio, sin necesidad de equipo especial y se pueden utilizar tanto en el medio urbano como rural.

Los resultados de este trabajo indican que un puntaje menor a 40 en la versión abreviada del examen cognoscitivo transcultural se asocia de manera independiente con un incremento en la mortalidad dentro de los siguientes dos años, confirmando hallazgos similares de otros estudios epidemiológicos. En este sentido, la evaluación cognoscitiva de tamizaje con el Examen Mínimo del Estado Mental se propone como un instrumento válido y efectivo para identificar de manera temprana y económica a pacientes con deterioro cognoscitivo. Un diagnóstico oportuno facilitaría la aplicación de medidas preventivas o terapéuticas más efectivas para reducir o controlar factores de riesgo modificables que puedan incidir en la mortalidad.

\section{REFERENCIAS}

1. Zúñiga Herrera E, García JE. El envejecimiento demográfico en México. Principales tendencias y características. Horizontes. 2008; 13:93-100.

2. Pérez Martínez VT. El deterioro cognitivo: una mirada previsora. Rev Cubana Med Gen Integr. 2005;21:1-2.

3. Petersen RC. Conceptual overview. En: Petersen RC. Mild cognitive Impairment. From aging to Alzheimer's disease. $1{ }^{a}$ ed. Nueva York: Editorial Oxford; 2003. Pp. 1-15.

4. Busch RM, Chelune GJ, Suchy Y. Using Norms in Neuropsychological Assessment of the Elderly. En: Attix DK, Welsh-Bohmer KA. Geriatric Neuropsychology Assessment and Intervention. 1. ${ }^{\mathrm{a}}$ ed. Nueva York: Editorial Guilford; 2006. Pp. 133-57.

5. Fletcher RH. Abnormality. En: Clinical Epidemiology: The essentials. 3. ${ }^{a}$ ed. Baltimore, Maryland: William Wilkins; 1996. Pp. 19-42.

6. Gauthier S, Touchon J. Mild cognitive impairment is not a clinical entity and should not be treated. Arch Neurol. 2005;62:1164-6.

7. Kave G, Heinik J. Issues to consider when using the new diagnosis of mild cognitive impairment. Isr Med Assoc J. 2005;7:732-5.

8. Winblad B, Palmer K, Kivipelto M, Jelic V, Fratiglioni L, Wahlund L-O, et al. Mild cognitive impairment-Beyond controversies, towards a consensus: Report of the International Working Group on Mild Cognitive Impairment. J Intern Med. 2004;256:240-6.

9. Mejía-Arango S, Miguel-Jaimes A, Villa A, Ruiz-Arregui L, Gutiérrez-Robledo LM. Dete- rioro cognoscitivo y factores asociados en adultos mayores en México. Salud Publica Mex. 2007;49:475-81.

10. Swan GE, Carmelli D, La Rue A. Performance on the digit symbol substitution test and 5year mortality in the Western Collaborative Group Study. Am J Epidemiol. 1995;141: 32-40.

11. Liu IY, LaCroix AZ, White LR, Kittner SJ, Wolf PA. Cognitive impairment and mortality: A study of possible confounders. Am J Epidemiol. 1990;132:136-43.

12. Deeg DJH, Hofman A, van Zonneveld RJ. The association between change in cognitive function and longevity in Dutch elderly. Am J Epidemiol. 1990;132:973-82.

13. Storandt M, Grant EA, Miller JP, Morris JC. Rates of progression in mild cognitive impairment and early Alzheimer's disease. Neurology. 2002;59:1034-41.

14. Tuokko H, Frerichs R, Graham J, Rockwood K, Kristjansson B, Fisk J, et al. Five-year follow-up of cognitive impairment with no dementia. Arch Neurol. 2003;60:577-82.

15. Frisoni GB, Fratiglioni L, Fastbom J, Viitanen M, Winblad B. Mortality in nondemented subjects with cognitive impairment: the influence of health-related factors. Am J Epidemiol. 1999;150:1031-44.

16. Gussekloo J, Westendorp RG, Remarque EJ, Lagaay AM, Heeren TJ, Knook DL. Impact of mild cognitive impairment on survival in very elderly people: cohort study. BMJ. 1997; 315:1053-4.
17. Batty GD, Deary IJ, Gottfredson LS. Premorbid (early life) IQ and later mortality risk: systematic review. Ann Epidemiol. 2007;17:278-88.

18. Fuentes-Cantú A, Ávila-Funes JA, GarcíaMayo E. Validez y confiabilidad del cuestionario de la ENASEM para la depresión en adultos mayores. Salud Publica Mex. 2007; 49:256-62.

19. Nguyen HT, Black SA, Ray LA, Espino DV, Markides KS. Cognitive impairment and mortality in older mexican americans. J Am Geriatr Soc. 2003;51:178-83.

20. Galindo-Villa Molina G, Balderas E. La evaluación neuropsicológica del anciano. Salud Mental. 2004;27(3):9-18.

21. Schaie KW, Willis SL, Caskie GI. The Seattle longitudinal study: relationship between personality and cognition. Neuropsychol Dev Cogn B Aging Neuropsychol Cogn. 2004;11: 304-24.

22. Arauz A, Alonso E, Rodríguez-Saldaña J Reynoso-Marenco M, Benitez IT, Mayorga $\mathrm{AM}$, et al. Cognitive impairment and mortality in older healthy Mexican subjects: a population-based 10-year follow-up study. Neurol Res. 2005;27(8):882-6.

23. Gutiérrez-Robledo LM, Villalpando-Berumen JM, García-Cruz JC, Reyes-Guerrero JA, Villa AR. Long-term survival related to cognitive performance in older adults treated at a tertiary hospital level. Gac Med Mex. 2009;145: 401-6.

24. Bosworth HB, Schaie KW, Willis SL. Cognitive and sociodemographic risk factors for mortality 
in the Seattle Longitudinal Study. J Gerontol B Psychol Sci Soc Sci. 1999;54:273-82.

25. Bruce ML, Hoff RA, Jacobs SC, Leaf PJ. The effects of cognitive impairment on 9-year mortality in a community sample. J Gerontol B Psychol Sci Soc Sci. 1995;50:289-96.

26. Gurland $\mathrm{H}$, Wilder D. Differences in rates of dementia between ethno-racial groups. En: Marin LG, Soldo BJ, eds. Racial and Ethnic Differences in the Health of Older Americans. Washington, DC: National Academy Press; 1997. Pp. 233-69.

27. Gurland BJ, Wilder DE. Relative rates of dementia by multiple case definitions, over two prevalence periods, in three cultural groups. Am J Geriatr Psychiatry. 1995;3:6-20.

28. Callahan CM, Hall KS, Hui SL, Musick BS, Unverzagt FW, Hendrie HC. Relationship of age, education, and occupation with dementia among a community-based sample of African Americans. Arch Neurol. 1996;53:134-40.
29. Stern Y, Gurland B, Tatemichi TK, Tang MX, Wilder D, Mayeux R. Influence of education and occupation on the incidence of Alzheimer's disease. JAMA. 1994;271:1004-10.

30. Bassuk SS, Wypij D, Berkman LF. Cognitive impairment and mortality in the communitydwelling elderly. Am J Epidemiol. 2000;151: 676-88.

31. Kelman HR, Thomas C, Kennedy GJ, Cheng J. Cognitive impairment and mortality in older community residents. Am J Public Health. 1994;84:1255-60.

32. Zelinski EM, Crimmins E, Reynolds $S$, Seeman T. Do medical conditions affect cognition in older adults? Health Psychol. 1998; 17:504-12.

33. Christensen $H$, Jorm AF, Henderson AS, Mackinnon AJ, Korten AE, Scott LR. The relationship between health and cognitive functioning in a sample of elderly people in the community. Age Ageing. 1994;23:204-12.
34. Lazcano-Ponce E, Esteve Fernández E, SalazarMartínez E, Hernández-Avila M. Estudios de cohorte. Metodología, sesgos y aplicación. Salud Publica Mex. 2000;42:230-41.

35. Fried LP, Kronmal RA, Newman AB, Bild DE, Mittelmark MB, Polak JF, et al. Risk factors for 5 -year mortality in older adults: the Cardiovascular Health Study. JAMA. 1998;279:585-92.

36. Fried LP, Tangen CM, Walston J, Newman $A B$, Hirsch C, Gottdiener J, et al. Frailty in older adults: evidence for a phenotype. J Gerontol A Biol Sci Med Sci. 2001;56:146-56.

Manuscrito recibido el 23 de octubre de 2009. Aceptado para publicación, tras revisión, el 7 de febrero de 2010.

ABSTRACT Objective. Determine the correlation between the low cognitive performance recorded in 2001 in the National Study on Health and Aging in Mexico (ENASEM) and the mortality reported in 2003 during the second phase of that same study.

\section{Cognitive performance and mortality in people over 50 in Mexico}

Key words
Methods. The subjects selected were people aged 50 or over who had completed the sections in the 2001 survey pertaining to cognitive exercises, health status, and functionality and assistance with daily activities. Cognitive performance was assessed in 2001 using an abridged version of the Cross-Cultural Cognitive Examination (scale: 0 to 80 points). In 2003, there was a follow-up to the interviewees, in which their survival or death was recorded.

Results. The groups of interviewees scoring less than 40 had a cumulative survival rate of $96.1 \%$, with a mean of 26.49 months of survival (CI 95\% 26.41-26.57), while those scoring 40 points or over had a cumulative survival rate of $98.7 \%$, with a mean of 28.76 months (CI 95\% 28.68-28.85) ( $\log$ Rank $\left.X^{2}=59,230 P<0,001\right)$. A score of less than 40 in the cognitive assessment was associated with a relative risk of death of 1.863 (CI: 95\% 1.30-2.65) in the multivariate analysis. Also associated with higher mortality was older age, self-reported diabetes, cancer, having smoked at some time, receiving assistance in at least one instrumental activity of daily living, and scoring higher on the depression scale.

Conclusions. A score of less than 40 in the abridged version of the Cross-Cultural Cognitive Examination was independently correlated with an increase in mortality within the next two years.

Aging; mortality; adult health; health of the elderly; mental health; activities of daily living; Mexico. 